\title{
CONTROLLING LIGHT BY LIGHT: OPTICAL MODULATION IN CHIRAL NEMATICS
}

\author{
M. SIERAKOWSKI* \\ Institute of Physics, Warsaw University of Technology \\ Koszykowa 75, 00-662 Warsaw, Poland
}

\begin{abstract}
Nonlinear optics of chiral liquid-crystalline media have been in scope of our interest for several years. Our previous observations in the subject had already been reported. This paper discusses a possibility of utilising the nonlinear optical properties of liquid crystals for practical purposes. We discuss an idea of "in line" intensity measurements of laser beam. The idea is based on two optical phenomena which are observed in liquid-crystalline media possessing a periodically stratified structure: nonlinear optical effect and selective light reflection. The effects start to appear by activating light intensities of the order of $\mathrm{MW} / \mathrm{m}^{2}$, so the method can be potentially applied at present to high-intensity light beams (corresponding laser power is approximately $0.1 \mathrm{~W}$ ), although there are now also prospects to lower this value significantly.
\end{abstract}

PACS numbers: 42.65.-k, 42.81.Wg, 42.70.Df

\section{Introduction}

Joining optical anisotropy and unusual sensitivity to many external agents liquid crystals (lc's) have been up to now extensively applied in variety of displays and optical actuators as well as sensors of numerous physical parameters, as temperature, pressure, mechanical stress, electric and magnetic field, etc. Nevertheless they still seem to offer new potential applications. The idea of using optical nonlinearity of lc for practical purposes was generally proposed previously [1], but, as far as we know, our work is the first experimental attempt to realise it. This paper considers in detail the use of reflected-probe-beam arrangement in combination with optical-fibre technique for "in line" monitoring high-intensity light beams. This particular application resulted directly from our earlier experiments in nonlinear optics of lc's [2,3]. We hope, it meets also some expectation in optical practice, because the users of lasers, especially in science, but also in industry, working in non-standard configurations, need in many cases to measure the beam intensity in a certain point of optical path. Indications of laser control unit are

*e-mail: Sierak@if.pw.edu.pl 
not adequate because the beam passing through a series of optical elements losses its initial energy. All the present "conventional" methods of light intensity measurements require splitting of the beam and putting a part of it to a meter. This part of optical energy is of course irretrievably lost and the propagating beam is attenuated. Although this attenuation is rather small and usually may be without importance, the splitting ratio depends on the beam polarisation and can change with it, introducing uncontrolled measurement error and perturbing polarisation relations of the outcoming light. In our conception we see a possibility to overcome the above inconveniences.

\section{Chiral nematics}

The age and the decade just pass since the discovery of lc's in 1888. The first observation was made in 1888 by German biologist Reinitzer and physicist Lehman in a periodically structured cholesterol derivative, which we now classify as chiral nematics (CHNs). Since then, many other types of lc's have been discovered, investigated in detail and also applied in many fields [4]. However, nonlinear optical phenomena in lc's have been the matter of the last two decades, since the high intensity lasers became accessible. As it is clearly shown in numerous monographs and papers [5, 6], two different types of lc's are organised in periodical structures: smectics and chiral nematics. A specific structural feature of CHNs are parallel molecular planes, in which long molecules of the species are unidirectionally oriented whereby, the molecular directions in the neighbour planes are slightly rotated to each other (Fig. 1). It makes a kind of helical structure where similar planes equally oriented are distanced by the helix pitch $p$, a characteristic value for the substance.

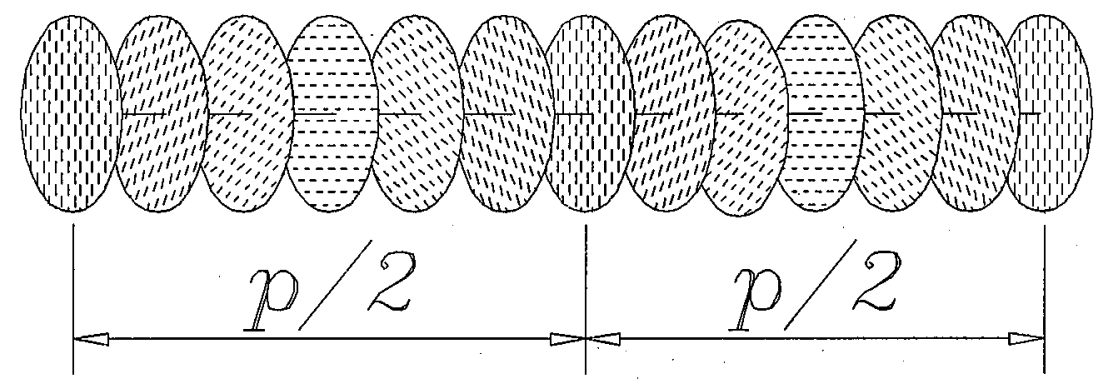

Fig. 1. Planar helical structure of chiral nematics. Dashes inside planes represent the average molecular orientation, $p$ is the helix pitch.

However, this $p$-value can be easily influenced by external agents as temperature, electric and magnetic fields and also by optical field as well. The structural periodicity of CHNs, i.e. the basic (equilibrium) value of the helical pitch can be varied by selecting composition of the substance; the pitch can be so matched to the light wavelength also in the visible range. The helical structure, optical anisotropy and easy structural deformation of CHNs evoke their unique optical properties, two of which are here of particular interest: 
- selective reflection of light

- structural optical nonlinearity.

Selective light reflection gives reflexes of light impinging a lc-layer, also in the visible spectrum, which resembles the röntgenographic diffraction in crystal lattices. The angle $\Theta_{B}$ of the constructive interference of light reflected from planar-oriented $\mathrm{CHN}$-structure giving maximum intensity can be derived from the typical Bragg formula [5]

$$
p \cos \Theta_{\mathrm{B}}=\lambda
$$

for the first order of diffraction ( $\lambda$ being light wavelength in the medium).

Optical nonlinearity generally means mutual interaction of a medium and propagating light. There are several mechanisms of the effect [6]. One of them, structural optical nonlinearity is a specific property of liquid crystals. The orientational mechanism of this kind of nonlinearity which appears as a feature of substances having collective molecular motion, together with the thermal one, bring lc's in the class of the strongest nonlinear materials. The structural nonlinearity occurs because the optical electric field of the propagating light wave tends to align the lc molecules along the $\boldsymbol{E}$-vector. If it is strong enough to influence the medium, then the changes introduced by light in the structure in turn affect back the light wave and give rise to a few spectacular optical phenomena. In the case of chiral nematics the optical field of light can influence both pitch of the helix and its orientation with respect to the layer plane. The first effect occurs by relatively high light intensities - of the order of $0.1 \mathrm{MW} / \mathrm{cm}^{2}$, while the second. can be already observed by 2-3 orders of magnitude lower fields. Moreover, recent reports [7] indicate a possibility to shift down apparently those values by adding to lc-layer a small amount of some (dye) dopants which probably involves another, new mechanism of optical nonlinearity based on photoinduced charge, known as Janossy effect.

\section{Principle of intensity measurement}

The proposed method of intensity measurement utilises interaction of propagating laser beam across CHN medium with its structure and uses selective reflection for the detection of the effect. The optical set-up is outlined in Fig. 2. The monitored intense laser beam ("argon pump laser" in the figure) propagates at normal incidence through the cell consisting of thin planar layer of CHN.

The optical field of the beam deforms locally the helix of lc. The second "probe" beam enlightens the deformation area at a glancing angle $\Theta$ close to the Bragg angle $\Theta_{B}$, therefore being strongly reflected to a detector. In this configuration any changes of helix tilt or its pitch induced by first (argon laser) beam will then influence reflection of the second (probe) beam. Its intensity is converted onto electrical signal by a photodiode. The photodiode signal will therefore vary with the monitored beam intensity.

\section{Experimental examination of the set-up}

The accomplishment of this idea is shown in Fig. 3. For practical reasons the probe-beam is carried by optical fibres. It allows us to reject parasite reflections 


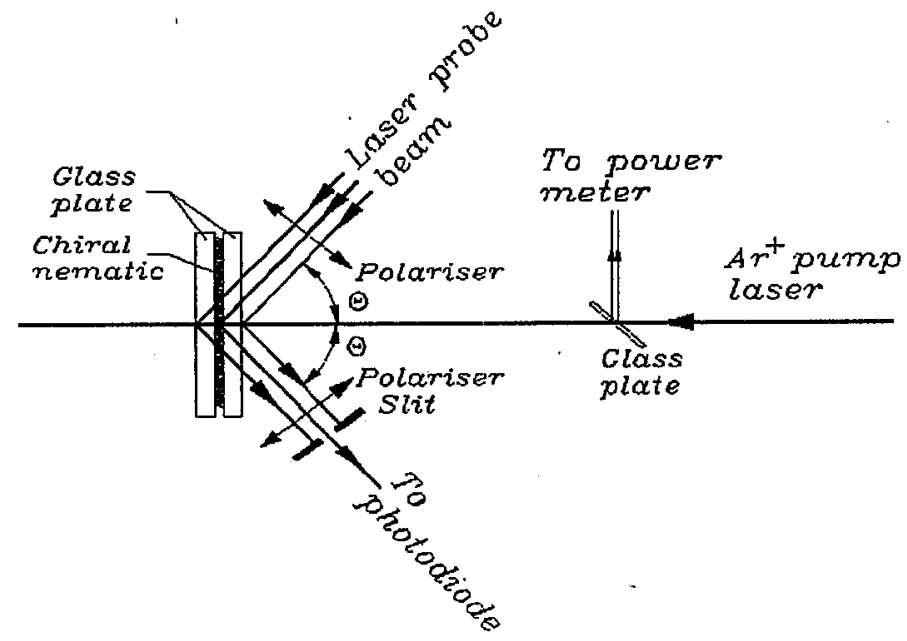

Fig. 2. Optical configuration for monitoring the argon laser intensity.

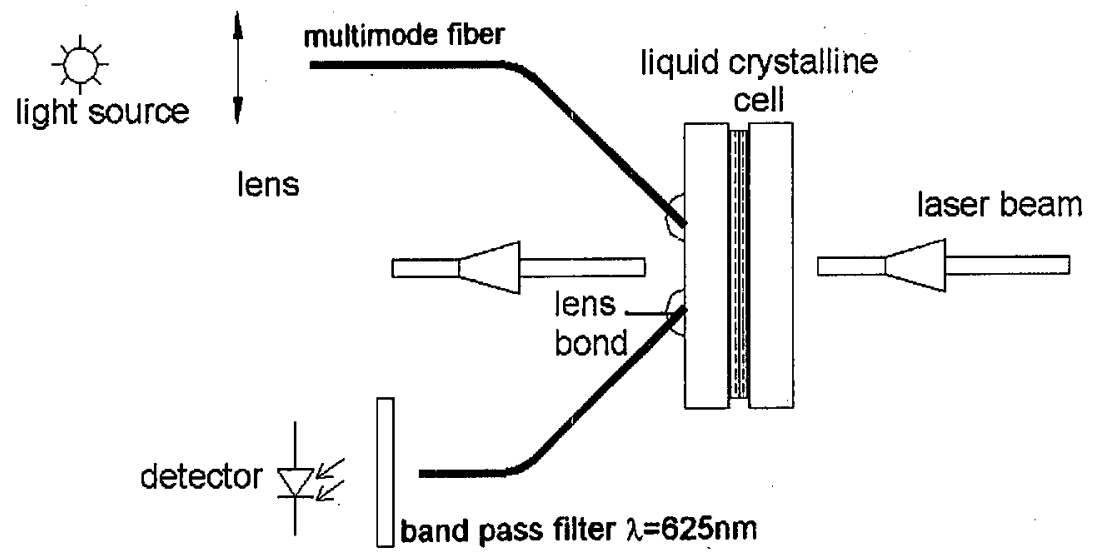

Fig. 3. Experimental set-up of the CHN light modulator.

from air/glass interfaces of the cell and helps to better define the desired reflection angle and makes the device more compact and stable.

Similarly, as a probe-beam source a conventional white-light lamp was used. This modification gives a more constructional flexibility because it accepts certain errors in angular arrangement of the device - there are always wavelengths in the lamp spectrum obeying selective reflection condition (1). However, in this case a reflected probe-beam under monitored laser illumination changes intensity and colours as well and a suitable filter in front of the photodetector is necessary. The geometry of the set-up was carefully adjusted to have good optical performance.

The photodiode signal recorded versus the intensity of the monitored laser is plotted in Fig. 4. The measurements are performed for chiral nematic mixture having the pitch value of $900 \mathrm{~nm}$ prepared on special order in Institute of 


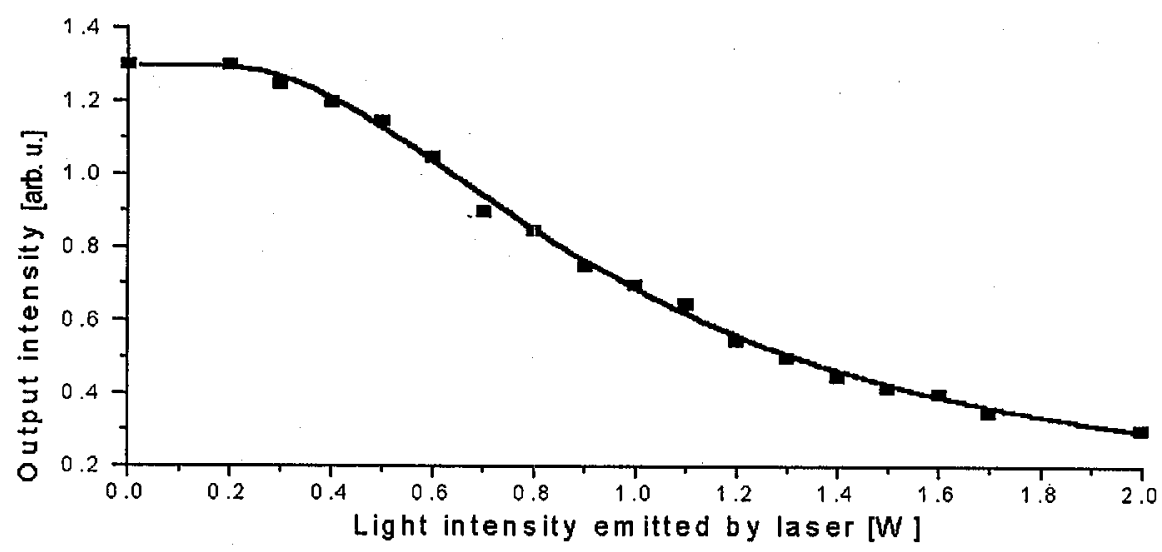

Fig. 4. Output-input characteristics of the modulator.

Physics of Warsaw Military Academy. The substance is thermally compensated to maintain a stable pitch value within $2 \mathrm{~nm}$ in the range $10 \div 30^{\circ} \mathrm{C}$ in order to exclude the thermal contribution in the structural nonlinearity. In the presented case the multi-mode optical fibres (200 $\mu \mathrm{m}$ core cladd-polymer) were used as probe-beam guides. The signal follows the beam intensity monotonically in the range of experimental laser power, giving direct output-input characteristics of the device. Clearly, this result could be considered as a first approach showing only the potential application of the effect to an intense-laser control. For practical use, properties of the set-up are to be carefully optimised in order to obtain a suitable performance, dynamic response, insensitivity on ambient influences (temperature), temporal stability, low perturbation of the measurand, etc. Distortions of the measured beam can arise in this optical configuration because of light losses and polarisation changes introduced by lc cell. Since absorption of $l c$ in the spectral range of practical interest is low and the lc-layer was thin $(25 \mu \mathrm{m})$, optical energy dissipation is mainly caused by back reflections from glass holder and from the lc layer. Since the first losses can be minimised by standard technological procedures (dielectric coatings), the second should be here shortly discussed. Reflections from the lc structure are directly related to selective reflection effect and circular dichroism of CHN used. Both of them cause maximal back reflexes in the "resonant" area, i.e. if $p \approx \lambda[5]$. In order to minimise them, this region must be avoided as the measured light is concerned. Outside the resonant range the transmitted beam can only suffer from polarisation plane rotation optically active lc layer. In principle this rotation can be compensated by a proper thickness matching lc layer, although in practice it can be troublesome because of the required mechanical precision. It is the matter of future work, whether this method of "in line" intensity measurements can ever become competitive with conventional ones in cost and performance.

\section{Summary}

In the paper a new concept of light intensity measurements of power lasers was presented. The method utilises the nonlinear optical effect in chiral liquid 
crystals. The preliminary results of experimental examination of the proposed method were demonstrated. Problems appeared by accomplishing the method were also defined.

\section{Acknowledgments}

The work was supported by the Committee for Scientific Research, grant no. 8 T11D 00412 .

\section{References}

[1] Tabiryan, T. Tschudi, T. Vogeler, B.Y. Zeldovich, in: Proc. 8th Annual Meeting IEEE Laser and Electro-Optics Soc., San Francisco 1995, Vol. 1, IEEE, p. 182.

[2] M. Sierakowski, M. Karpierz, Thang-Long Do, M. Roszko, Mol. Cryst. Liq. Cryst. 282, 139 (1996).

[3] M. Sierakowski, M. Karpierz, Mol. Cryst. Liq. Cryst 320, 149 (1998).

[4] see for example: Proc. VIIth Int. Topical Meeting on Optics of Liquid Crystals, Heppenheim (Germany) 1997, Mol. Cryst. Liq. Cryst 320, (1998).

[5] see for example: S. Chandrasekhar, Liquid Crystals, Cambridge University Press, London 1977.

[6] I.C. Khoo, S.T. Wu, Optics and Nonlinear Optics of Liquid Crystals, World Scientific, Singapore 1993.

[7] I.C. Khoo, B.D. Guenther, M.V. Wood, M.Y. Shih, P.H. Chen, in: Liquid Crystals II, Ed. I.C. Khoo, in series Proc. SPIE, Vol. 3475, 1998, p. 88. 